\title{
Effect of electron beam irradiation on photoluminescence properties of thioglycolic acid (TGA) capped CdTe nanoparticles
}

\section{Chethan Pai ${ }^{1}$, M. P. Joshi ${ }^{\star}$, S Raj Mohan², T. S. Dhami' ${ }^{2}$ Jayakrishna Khatei ${ }^{3}$, K S Koteshwar Rao ${ }^{3}$, L. M. Kukreja ${ }^{2}$, Ganesh Sanjeev ${ }^{1}$}

\author{
${ }^{1}$ Microtron Centre, Department of Studies in Physics, Mangalore University, Mangalagangotri, India \\ ${ }^{2}$ Laser Materials Processing Division, Raja Ramanna Centre for Advanced Technology, Indore, India \\ ${ }^{3}$ Department of Physics, Indian Institute of Science, Bangalore, India
}

*Corresponding author. Tel. (+91) 731 2488353; E-mail: mukesh@rrcat.gov.in

Received: 29 January 2012, Revised: 17 February 2012 and Accepted: 19 February 2012

\section{ABSTRACT}

Irradiation effects of $8 \mathrm{MeV}$ electrons on photoluminescence properties of thioglycolic acid (TGA) capped CdTe quantum Dots (QD) are presented. Steady-state and time-resolved photoluminescence (PL) spectroscopy were used for anlayzing PL properties of both irradiated and unirradiated quantum dots. The Photoluminescence peak, intensity and lifetimes were found to vary with dose. At lower doses (up to 5kGy), they were found increasing and at higher doses (up to 20kGy) it decreased. The PL peak position also shifted toward low energy and broadened with increase of dose. Initial increase in PL intensity (upto $5 \mathrm{kGy}$ ) is due to passivation of surface defects leading to high radiative recombination. At higher doses the damage of capping layer takes place leading to aggregation effects. Copyright $@ 2013$ VBRI press.

Keywords: Nanoparticles, electron beam irradiation, photoluminescence, $\mathrm{CdTe} / \mathrm{CdS}$ core/shell structure.
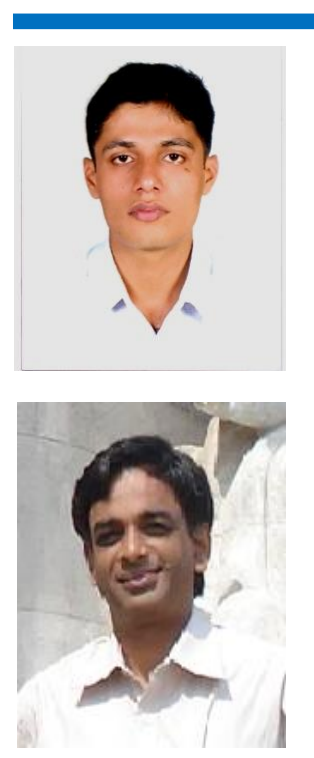

Chethan Pai $\mathbf{S}$ is presently working as Research Scholar (2008- Present) at Mangalore University, Karnataka. His research topic include influence of electron beam irradiation on nanomaterials physical and optical properties.

M. P. Joshi is presently working as Scientific Officer (1988- Present). He is actively involved in $\mathrm{R} \& \mathrm{D}$ of nanocomposite photonic materials for applications such as photovoltaic, NLO, $\mathrm{THz}$ sources and detectors.

\section{Introduction}

Semiconductor nanoparticles or quantum dots (QDs) have received much attention due to their unique size dependent optoelectronic properties and are currently of much interest for their technological applications. QDs of Cadmium Telluride (CdTe), due to its size dependent tunable photoluminescence (PL) property, have found numerous applications in lasers, sensors, solar cells and bio-labelling etc. It is known that, in general, the emission properties and photo-stabilities of QDs are strongly affected by the surface properties due to higher surface to volume ratio. The factors which govern the emission properties are surface non-stoichiometry and unsaturated bonds due to improper surface passivation. This has made surface passivation using organic and inorganic molecules as one of the main domains in quantum dot research. To achieve improved spectral properties of QDs, including that of CdTe, the concept of core/shell structure QDs has been shown as one of the effective means of surface passivation. Here effort is made for epitaxial growth of an inorganic shell with a broader band gap on the surface of luminescence nanoparticles. Most of the studies for core-shell structure QDs focus on employing chemical or photochemical methods. Hot-injection organometallic approaches have been employed successfully but these QDs are usually 
synthesized under rigorous experimental conditions. Moreover, the QDs are often not dispersible in aqueous media [15-17] which seriously limits their applications e.g. in bio-labelling. Some research groups have observed surface passivation of thiol-capped water soluble CdTe QDs after exposing them to UV radiation [1,2]. Using ionisation radiation e.g. electron beams, the synthesis of core/shell $\mathrm{CdTe} / \mathrm{CdS}$ in water or in aqueous polymers is also considered as promising tool for various applications [8]. However, studies on the use of electron beam irradiation on controlling the surface properties of QDs are very sparse. Such studies are not only important for the synthesis of core/shell QDs but it also provides insight into the mechanism of formation of core/shell nanostructure $[\mathbf{5}$, 20-21]. In this paper we investigate the dose dependent effects of $8 \mathrm{MeV}$ electron beam irradiation on photoluminescence (PL) properties of CdTe QDs. Study of photoluminescence properties of nanoparticles provides an efficient means of investigating changes in surface properties (the passivation) and/or changes in shape and size of nanoparticles. A study has been undertaken to investigate use of electron beams for the synthesis of coreshell CdTe-CdS QDs. Hydrothermal synthesis route was employed to prepare aqueous colloidal solution of thioglycolic acid (TGA) capped CdTe QDs. Steady-state and time-resolved PL investigations of irradiated QDs suggests initial increase in PL intensity (for dose upto $5 \mathrm{kGy}$ ) is due to passivation of surface defects leading to high radiative recombination. At higher dose (up to 20 $\mathrm{kGy}$ ) the damage of TGA capping layer takes place leading to aggregation effects and defect formation leading to decrease in PL intensity.

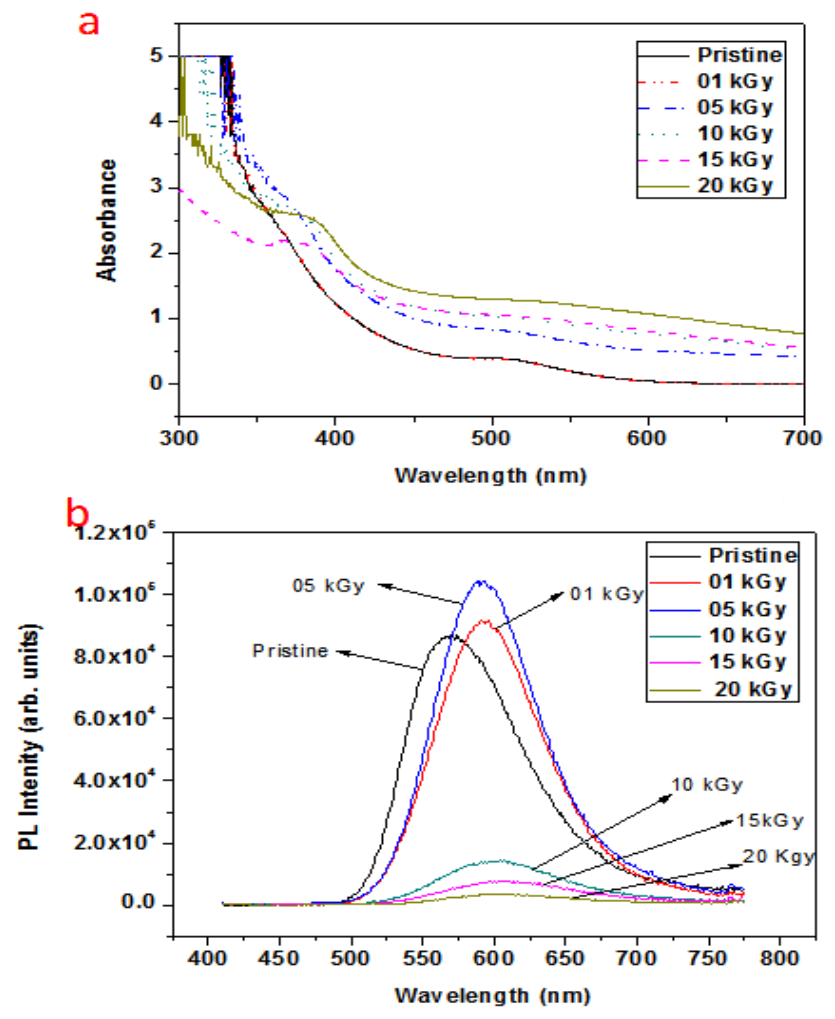

Fig. 1. (a) Absorption spectra of pristine and irradiated samples with doses from $1 \mathrm{kGy}$ to $20 \mathrm{kGy}$. (b) Emission spectra of pristine and irradiated samples with does from $1 \mathrm{kGy}$ to $20 \mathrm{kGy}$.

\section{Experimental}

Hydrothermal method was used for the synthesis of thioglycolic acid (TGA) capped CdTe QDs. Trisodium citrate dihydrate $(100 \mathrm{mg})$, Sodium Tellurite $(0.01 \mathrm{~mol} / \mathrm{L}$, $4 \mathrm{~mL}$ ), and Sodium borohydride (50 mg) were successively added to $4 \mathrm{~mL}$ of Cadmium Chloride solution $\left(\mathrm{CdCl}_{2}, 0.04\right.$ $\mathrm{mol} / \mathrm{L}$ ) and diluted by adding $42 \mathrm{~mL}$ ultra-pure water. TGA was added to the as prepared solution. The solution was autoclaved in stainless steel autoclave with teflon liner at $160{ }^{\circ} \mathrm{C}$ for $45 \mathrm{~min}$. For electron beam irradiation studies we used Microtron facility at Mangalore University, India. The samples were taken in micro tubes and exposed to 8 $\mathrm{MeV}$ electrons at a distance of $30 \mathrm{~cm}$ from the beam exit port. Electron doses used were ranging from $1 \mathrm{kGy}$ to 20 kGy. The details of the facility are mentioned elsewhere [19]. Optical absorption were characterised using a Shimadzu UV-3101PC double beam absorption spectrophotometer. Edinburgh FLS 920 spectrofluorometer was used for both the steady state and the time resolved PL lifetime measurements. For lifetime measurements a time correlated single photon counting (TCSPC) technique was used with picosecond LEDs (320nm excitation wavelength, pulse width $\sim 750 \mathrm{ps}$ and $1 \mathrm{MHz}$ rep rate) as excitation source.

\section{Results and discussion}

$U V$-Vis spectroscopy

Absorption spectrum provides very useful information on the after-effects of electron beam irradiation. Fig. 1a show absorption spectrum of unirradiated pristine and irradiation dose dependent CdTe QDs in water. Pristine sample shows absorption peak at $507 \pm 2 \mathrm{~nm}$ which corresponds to band gap energy of $2.44 \pm 0.01 \mathrm{eV}$. Average size of nanoparticles estimated from absorption data was $\sim 2 \mathrm{~nm}$, which is well below exciton Bohr radius for CdTe $\sim 7 \mathrm{~nm}$. Irradiated samples shows red shift in absorption peak with respect to pristine and the shift in absorption peak was $10 \pm 2 \mathrm{~nm}$ after dose of $20 \mathrm{kGy}$. Red shift in UV-Visible absorption spectrum with dose can be attributed to increase in nanoparticles size. At higher dose we also observe additional features in the absorption spectra around 400nm (very prominent for 15 and $20 \mathrm{kGy}$ dose) and also overall increase of absorbance over entire spectral range. The additional absorption could be due to damage of capping layer leading to formation of UV absorbing byproducts or formation wide bandgap CdS shell on CdTe core [18]. Simultaneous increase in absorbance over the entire spectral range of absorption spectrum could be due to aggregation of QDs. Thus formed aggregates contribute transmission loss due to Rayleigh scattering and hence increase in absorbance with dose [2]. This is also supported by the observation of black precipitate in clear solution for higher dose regime.

\section{Steady state and time resolved emission spectroscopy}

Most pronounced effects of electron irradiation were observed in steady state and time resolved PL spectra. Fig. 1b shows the steady state PL spectra of pristine and irradiated samples when excited at $320 \mathrm{~nm}$. Considerable amount of red shift and broadening of excitonic emission 
was observed with increase in dose. The PL intensity also changes with increase in electron dose. Upto 5kGy dose the PL intensity increases while at further higher dose the PL intensity decreases drastically. These changes in PL intensity and emission wavelength with dose are shown in Fig. 2.

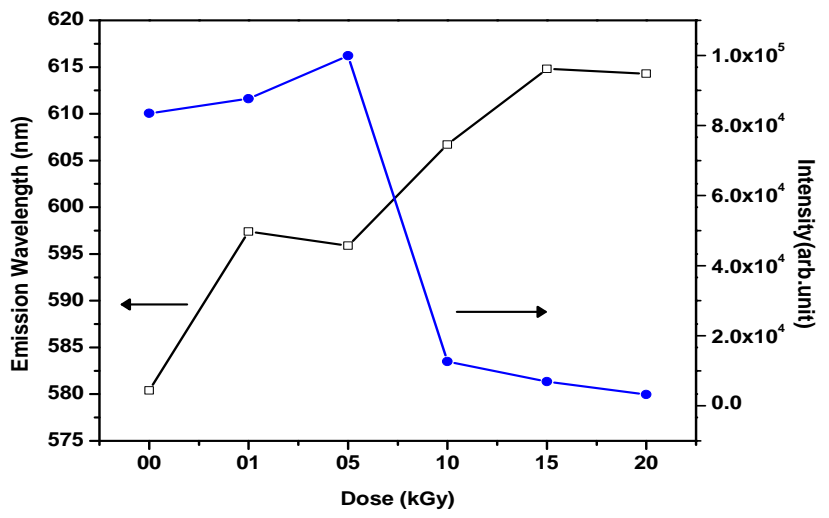

Fig. 2. Variation of emission intensity and corresponding emission wavelengths with respect to irradiation doses from $1 \mathrm{kGy}$ to $20 \mathrm{kGy}$.
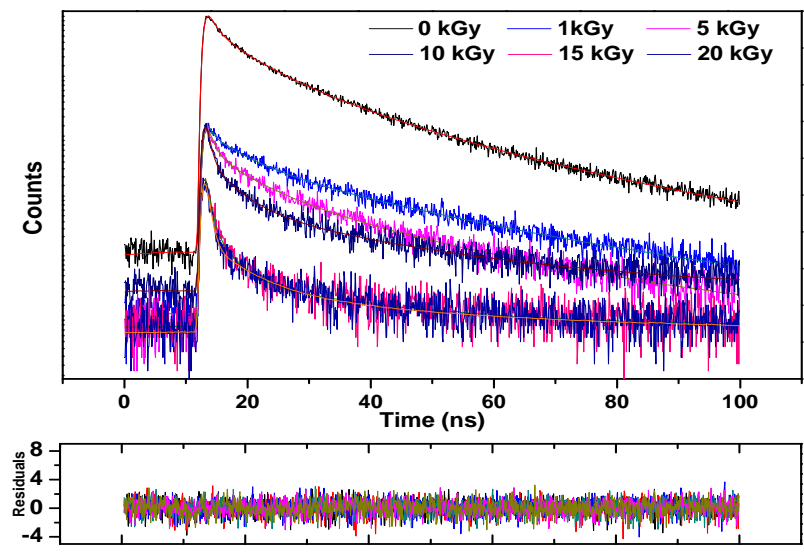

Fig. 3. Emission decay curves for various doses, ranging from $1 \mathrm{kGy}$ to 20 kGy. Residue error (the $\chi^{2}$ values) for each decay curve fitted with triple exponential function is also shown in panel below.

To gain further insight into the possible reasons for PL changes, we made PL lifetime measurements. Fig. 3 shows dose dependent emission decay curves monitored at peak emission wavelength. Each decay curve was fitted with triple exponential function,

$I(t)=A_{1} e^{-t / \tau_{1}}+A_{2} e^{-t / \tau_{2}}+A_{3} e^{-t / \tau_{g}}$

where A's and $\tau$ 's are pre-exponential factors and decay times respectively. Average PL lifetime was obtained using weighted average,

$\tau_{\text {avg }}=\frac{\sum_{i} A_{i} \tau_{i}^{2}}{\sum_{i} A_{i} \tau_{i}}$

Fitted decay parameters and estimated average PL lifetime for each dose is shown in Table 1. We observed that average life time increases initially upto $5 \mathrm{kGy}$ dose and then decreases with further dose. A strong correlation can be seen between variation of emission intensity and average lifetime with dose.

Table 1. Triple exponential decay parameter used for fitting PL lifetime data. Average lifetime $\tau_{\mathrm{av}}$ was obtained using weighted average, $\sum_{\mathrm{i}} A_{\mathrm{i}} \tau_{\mathrm{i}}^{2}$

$\frac{\sum_{i} A_{i} \tau_{i}}{\sum_{i} A_{i} \tau_{i}}$, where $A_{i}$ 's and $\tau_{i}$ 's are decay amplitudes and decay times respectively.

\begin{tabular}{|c|c|c|c|c|}
\hline Dose (KGy) & $\tau_{1}$ & $\tau_{2}$ & $\tau_{3}$ & $\begin{array}{c}\text { Average } \\
\text { lifetime } \tau_{\mathrm{av}} \text { in } \\
\mathrm{ns}\end{array}$ \\
\hline \multirow{3}{*}{ Pristine } & $1.3244 \mathrm{~ns}$ & $9.0321 \mathrm{~ns}$ & 32.3461 & \\
\hline & $\mathrm{A}_{1}=0.045$ & $\mathrm{~A}_{2}=0.038$ & $\mathrm{~A}_{3}=0.031$ & \\
\hline & $\operatorname{Rel} \%=4.1 \%$ & $\operatorname{Rel} \%=24.51 \%$ & $\operatorname{Rel} \%=71.32 \%$ & 25.33768 \\
\hline \multirow{3}{*}{1} & $0.5696 \mathrm{~ns}$ & $7.1832 \mathrm{~ns}$ & 38.5377 & \\
\hline & $\mathrm{A}_{1}=0.066$ & $\mathrm{~A}_{2}=0.034$ & $\mathrm{~A}_{3}=0.043$ & \\
\hline & $\operatorname{Rel} \%=1.94 \%$ & $\operatorname{Rel} \%=12.64 \%$ & $\operatorname{Rel} \%=85.42 \%$ & 33.41809 \\
\hline \multirow{3}{*}{5} & $1.0312 \mathrm{~ns}$ & $8.2444 \mathrm{~ns}$ & $35.118 \mathrm{~ns}$ & \\
\hline & $\mathrm{A}_{1}=0.13$ & $\mathrm{~A}_{2}=0.031$ & $\mathrm{~A}_{3}=0.017$ & \\
\hline & $\operatorname{Rel} \%=2.59 \%$ & $\operatorname{Rel} \%=13.06$ & $\operatorname{Rel} \%=75.35 \%$ & 23.52531 \\
\hline \multirow{3}{*}{10} & $0.5942 \mathrm{~ns}$ & $3.3891 \mathrm{~ns}$ & $31.6545 \mathrm{~ns}$ & \\
\hline & $\mathrm{A}_{1}=0.130$ & $\mathrm{~A}_{2}=0.031$ & $\mathrm{~A}_{3}=0.017$ & \\
\hline & $\operatorname{Rel} \%=9.90 \%$ & $\operatorname{Rel} \%=19.53 \%$ & $\operatorname{Rel} \%=70.57 \%$ & 24.20218 \\
\hline \multirow{3}{*}{15} & $0.5401 \mathrm{~ns}$ & $3.3891 \mathrm{~ns}$ & $26.0030 \mathrm{~ns}$ & \\
\hline & $\mathrm{A}_{1}=0.151$ & $\mathrm{~A}_{2}=0.035$ & $\mathrm{~A}_{3}=0.010$ & \\
\hline & $\operatorname{Rel} \%=18 \%$ & $\operatorname{Rel} \%=26.16 \%$ & $\operatorname{Rel} \%=55.84 \%$ & 15.6618 \\
\hline \multirow{3}{*}{20} & $0.4356 \mathrm{~ns}$ & $3.7674 \mathrm{~ns}$ & $27.0626 \mathrm{~ns}$ & \\
\hline & $\mathrm{A}_{1}=0.210$ & $\mathrm{~A}_{2}=0.023$ & $\mathrm{~A}_{3}=0.009$ & \\
\hline & $\operatorname{Rel} \%=21.68 \%$ & $\operatorname{Re} 1 \%=20.75 \%$ & $\operatorname{Rel} \%=57.57 \%$ & 16.4997 \\
\hline
\end{tabular}

The increase in PL intensity with electron dose could be due to several reasons. Similar enhancements of PL intensity in CdTe QDs have been reported when QDs are either exposed to UV light or under environments of different $\mathrm{pH}$ [5]. Bao et al. [5] observed a similar enhancement in TGA capped CdTe PL intensity after prolonged UV light irradiation. They also observed the dissociation of thioglycolic acid into sulphur ion and formation $\mathrm{CdS}$ shell on CdTe core on prolonged UV irradiation. Similar explanation can be proposed with electron beam. Therefore the most possible explanation for the observed increase in PL intensity and average decay time for radiation dose less than $5 \mathrm{kGy}$ is the passivation of the QD surface. In the solution of as prepared CdTe, apart from CdTe QDs, there are excessive thioglycolic acid and cadmium ions. Also, the core surface of as formed TGA capped QDs of CdTe may still have sufficient surface defects in the form of dangling bonds. The low dose of electron beam essentially passivates those surface states which are not blocked by the capping agent. Electron irradiation dissociates thioglycolic acid and releases sulphur ion as in the case of UV light irradiation. Thus sulphur ion react with cadmium ion on CdTe QD's surface and form CdS layer on CdTe. The CdS layer effectively passivates surface dangling bond and prevent exciton being trapped into surface states [5]. This significantly reduces the non radiative contribution to the exciton decay and lead to enhancement in PL intensity. Slight increase in decay lifetime for the same dose level can be attributed to decrease in surface trap states which act as hole traps [2]. This leads to decrease in non-radiative channel for exciton decay. Another possible reason for PL intensity enhancement could be the formation of $\mathrm{CdTe}_{\mathrm{x}} \mathrm{S}_{1-\mathrm{x}}$ alloy which has wider band gap compared to CdTe [7]. This 
possibility can be ruled out because we also observe slight red-shift of emission peak as well. In the case of alloy formation one would observed blue-shift of emission peak. The slight red-shift in emission peak at low dose also suggests increase in QD size. Since there is detachment of TGA group due to electron beam irradiation, the process of aggregation can therefore begin together with surface passivation process.

At higher dose the red-shift of PL peak is seen more pronounce together with decrease on PL intensity. PL decay time also decreases substantially at higher dose. Most obvious explanation for decrease in PL intensity and average lifetime for radiation dose more than $5 \mathrm{kGy}$ is due to formation of non-emissive byproducts and/or defects at the core surface. Since irradiation is carried out in water environment therefore oxidation of core surface can not be ruled out. Similar decrease in PL intensity for CdTe QDs has been reported when QDs are kept in oxidative environments [JPC B, 111, 12012, 2007]. Since continuous and systematic red-shift of PL peak is observed till 20kGy, the average size of QDs can be considered to keep increasing with dose (similar observations of size increase with dose are made from absorption data shown in Fig. 1a. The rise in absorbance values over the entire spectral range is primarily due to increase in Rayleigh scattering from large sized particles). Due to increase in defect formation on the core surface the trapping of hole or electron get prominent and subsequently a decrease in radiative recombination of excitation is expected. Similar observation can be made for PL lifetime data which decreases strongly with further increase of dose. There is one order of magnitude decrease in PL decay lifetime after the irradiation of QDs to $20 \mathrm{kGy}$. This decrease in average lifetime of is due to an efficient transfer of hole or electron from QDs into surrounding trap states.

The analysis of PL properties provides sufficient indication of dose level required for efficient passivation of QD surface or core-shell QDs for improved PL properties. The method of electron beam irradiation may be considered as an efficient means of processing QDs for obtaining stable and highly luminescent QDs for various applications. Further investigations on irradiated products are needed to ascertain the formation of $\mathrm{CdS}$ shell on CdTe core.

\section{Conclusion}

In this paper the effect of electron irradiation on TGA capped CdTe colloidal QDs was investigated using photoluminescence spectroscopy. In relatively low dose regime the increase in PL intensity and PL life time indicates further passivation of surface of CdTe QDs. The nature of passivation could be due to possible formation of $\mathrm{CdS}$ shell on $\mathrm{CdTe}$ core. At relatively higher doses regime the red-shift PL intensity suggest damage of capping layer leading to aggregates of QDs. Reasons for the decrease in PL at higher dose can be attributed to defect formation on the surface of CdTe QDs.

\footnotetext{
Acknowledgements

One of the authors (CP) thanks BRNS, DAE, Govt. of India for providing financial assistance. Authors thank research scholars and staff of Microtron Centre for their help and support during the course of this work.
}

Reference

1. Byrne, S. J.; Corr ,S. A.; Tatsiana, Y, Rakovich.; Yurii K, Gun, ko.; Yury, P, Rakovich.; Donegan, John, F.; Siobhan, Mitchell. ; Yuri Volkov. J. Mater. Chem. 2006, 16, 2896.

DOI: $\underline{10.1039 / \mathrm{b} 605333 \mathrm{e}}$

2. Trotzky, S.; Kolny, Olesiak, J.; Falke ,S, M.; Hoyer,T.; Lienau,C.; Tuszynski, W.; Parisi J. J. Phys. D: Appl. Phys. 2008, 41, 102004. DOI: $10.1088 / 0022-3727 / 41 / 10 / 102004$

3. Venkatram .; Reddeppa, N.; Sathyavathi T, R.; Umananda, Bhatta, M.; Satyam, P,V.; Narayana, Rao, D. Nucl. Instr. Meth. Phys. Res. B. 2008, 266, 1816. DOI: $10.1016 / \mathrm{j} . \mathrm{nimb} .2008 .01 .026$

4. Shailesh , Sharma, N.; Himani ,Sharma.; Gurmeet, Singh.; Shivaprasad S,M. Nucl. Instr. Meth. Phys. Res. B. 2006, 244, 86. DOI:10.1016/j.nimb.2005.11.014

5. Haobo, Bao.; Yanjun, Gong.; Zhen, Li.; Mingyuan, Gao. Chem. Mater. 2004, 16, 3853.

DOI: $10.1021 / \mathrm{cm} 049172 \mathrm{~b}$

6. Haesselbarth, A.; Eychmueller, A.; Eichberger, R.; Giersig, M.; Mews, A.; Weller. H. J. Phys. Chem. 1993, 97, 5333.

DOI: $10.1021 / \mathrm{j} 100122 \mathrm{a} 026$

7. Zhenyu ,Gu.; Lei, Zou.; Zheng, Fang.; Weihong, Zhu.; Xinhua, Zhong. Nanotechnology. $2008,19,135604$.

DOI: $10.1088 / 0957-4484 / 19 / 13 / 135604$

8. Yun, Lei.; Haiyang ,Tang.; Chunjiao, Zhou.; Tingting, Zhang.; Meifu, Feng.; Bingsuo, Zou. J. of Lumin. 2008, 128, 277. DOI: $10.1016 /$ j.jlumin.2007.07.011

9. Istvan , Robel.; Vaidyanathan, Subramanian.; Masaru, Kuno.; Prashant ,V, Kamat. J. Am. Chem. Soc. 2006, 128, 2385. DOI: $10.1021 / \mathrm{ja} 056494 \mathrm{n}$

10. Nozik, A,J. Physica E. 2002, 14, 115. DOI: $\underline{10.1016 / S 1386-9477(02) 00374-0}$

11. Kevin, Coakley, M.; Michael, McGehee, D. Chem. Mater. 2004, 16 , 4533. DOI : $10.1021 / \mathrm{cm} 049654 \mathrm{n}$

12. Kwong C,Y.; Choy H. W. C.; Djurišić, A. B.; Chui, P, C.; Cheng K. W., Chan ,W. K. Nanotechnology. 2004, 15,1156. DOI: $10.1088 / 0957-4484 / 15 / 9 / 008$

13. Swadeshmukul, Santra.; Heesun, Yang.; Paul, Holloway, H.; Jessie, Stanley, T.; Robert, A. Mericle. J. Am. Chem. Soc. 2005,127,1656. DOI: $10.1021 / \mathrm{ja} 0464140$

14. Cheng-An J, Lin.; Tim, Liedl.; Ralph, Sperling,A.; María, T.; Fernández,Argüelles.; Jose, Costa-Fernández, M.; Rosario, Pereiro.; Alfredo, Sanz-Medel.; Walter, H, Chang.; Wolfgang J. Parak. J. Mater. Chem. $2007,17,1343$.

DOI : $10.1039 / \mathrm{B} 618902 \mathrm{D}$

15. Margaret, A. Hines .; Philippe Guyot-Sionnest. J. Phys. Chem. 1996, $100,468$.

DOI: $10.1021 / \mathrm{jp} 9530562$

16. Renguo, Xie.; Ute, Kolb.; Jixue, Li.; Thomas, Basche.; Alf, Mews. J. Am. Chem. Soc. 2005,127, 7480 . DOI $: 10.1021 / \mathrm{ja} 042939 \mathrm{~g}$

17. Nikolai, Gaponik.; Dmitri ,V.; Talapin.; Andrey ,L.; Rogach.; Kathrin, Hoppe.; Elena, V.; Shevchenko.; Andreas Kornowski.; Alexander Eychmuller.; Horst Weller. J. Phys. Chem. B. 2002,106, 7177.

DOI: $10.1021 / \mathrm{jp} 025541 \mathrm{k}$

18. Siddappa, K.; Ganesh.; Balakrishna K, M.;. Ramamurthi S, S.; Soni H, C.; Shrivastava, P.; Sheth ,Y .; Hemnani, R. Radiat. Phys. Chem. 1998, $51,441$.

19. Ma, J.; Ji-Yao, Chen.; Guo, J.; Wang, C , C.; Yang W. L.; Xu, L.; Wang, P. N. Nanotechnology. 2006,17, 2083-2089. DOI: $10.1088 / 0957-4484 / 17 / 9 / 002$

20. Hui, Peng.; Lijuan Zhang.; Christian, Soeller.; Jadranka.; TravasSejdic. J. of Lumin. 2007,127, 721. DOI:10.1016/j.jlumin.2007.04.007
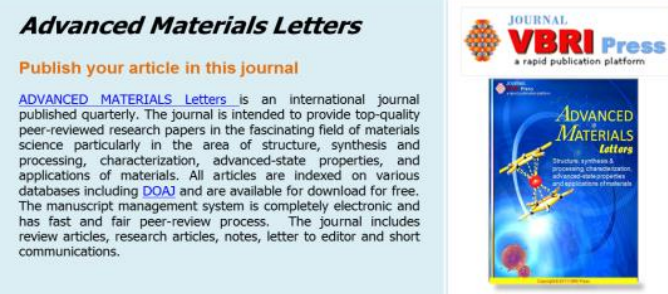